\title{
A Novel Hybrid Binarization Technique for Images of Historical Arabic Manuscripts
}

\author{
Aboul Ella HASSANIEN ${ }^{1}$, Mohamed ABDELFATTAH ${ }^{2}$, Khaled M. AMIN ${ }^{3}$, Sherihan MOHAMED ${ }^{2}$ \\ ${ }^{1}$ Faculty of Computers and Information, \\ Cairo University, Egypt, \\ aboitcairo@gmail.com. \\ ${ }^{2}$ Faculty of Computers and Information, \\ Mansoura University, Egypt. \\ ${ }^{3}$ Faculty of Computers and Information, \\ Menofia University, Egypt.
}

Abstract: In this paper, a novel binarization approach based on neutrosophic sets and sauvola's approach is presented. This approach is used for historical Arabic manuscript images which have problems with types of noise. The input RGB image is changed into the NS domain, which is shown using three subsets, namely, the percentage of indeterminacy in a subset, the percentage of falsity in a subset and the percentage of truth in a subset. The entropy in NS is used for evaluating the indeterminacy with the most important operation " $\lambda$ mean" operation in order to minimize indeterminacy which can be used to reduce noise. Finally, the manuscript is binarized using an adaptive thresholding technique. The main advantage of the proposed approach is that it preserves weak connections and provides smooth and continuous strokes. The performance of the proposed approach is evaluated both objectively and subjectively against standard databases and manually collected data base. The proposed method gives high results compared with other famous binarization approaches.

Keywords: Document image binarization, Historical manuscript image, Neutrosophic theory, Pixel classification.

\section{Introduction}

Libraries and archives in the world store a huge number of old and historically important manuscripts and documents. These historical documents accumulate a significant amount of human heritage over time [1]. Digital images of historical documents typically suffer from various degradations due to; uncontrolled storage conditions, ageing [2]. The main degradations are; non-uniform illumination, strain, smears, bleeds- through, faint characters, shadow $[1 ; 2 ; 3]$.

The binarization process is a key step in all document image processing workflows. It switches an image into bi-level form in such way that the background information is represented by white pixels and the foreground by white ones [3;4]. Although the process of document image binarization has been studied for many years ago, thresholding of historical document images is still a challenging problem due to the complexity of the images and the above mentioned degradations. Moreover, binarization of ancient Arabic manuscripts has extra problems such as; decorations, diacritics or characters written in multiple colors [2]. Figure 1 shows examples of degraded historical Arabic manuscript images.

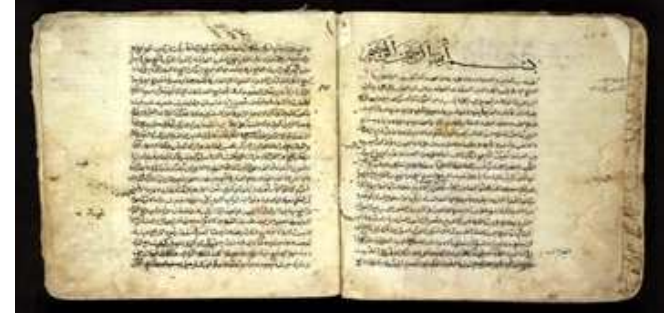

a) Dirty document with spots, stains, smears or smdges

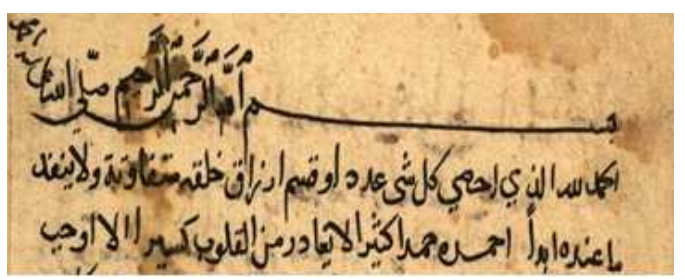

b) Ink wet characters visible both sides

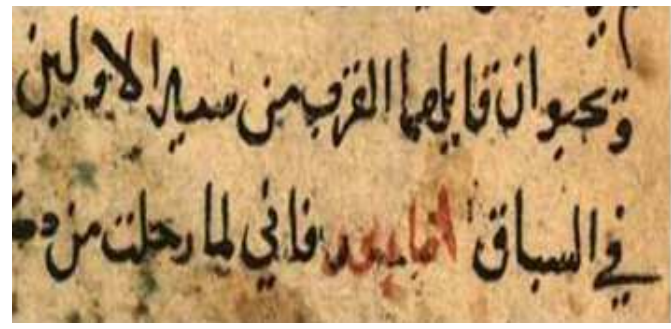

c) Broken characters, light handwriting 


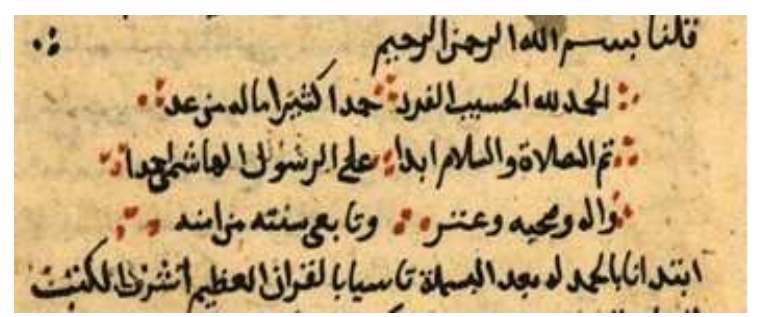

d) Documents with poor quality paper

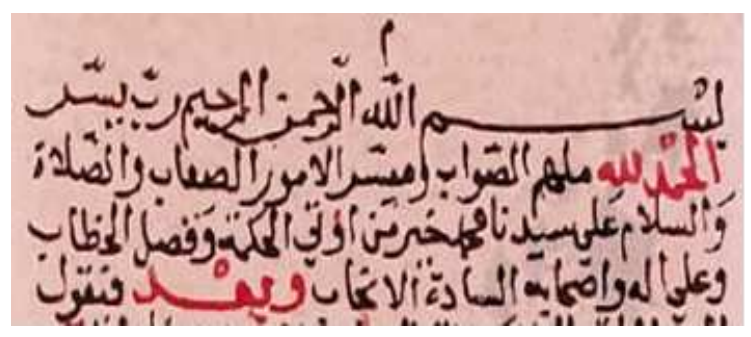

e) Multicolored, background

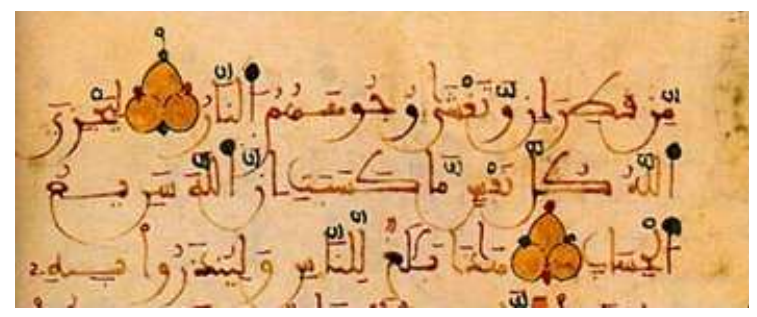

f) Poor contrast between

foreground and background

Figure 1. Examples of manuscript images containing multi-colored text lines with different degradations $[5 ; 6]$.

In this paper, a new hybrid algorithm for binarization of degraded Arabic manuscript image is proposed. It combines the famous adaptive algorithm of Sauvola's (8) and a NS binarization algorithm of [9] into a hybrid one. Neutrosophic set (NS) approach is quite new and have been useful for various image processing tasks such as segmentation, thresholding, and denoising [7]. Experimental results proves that the proposed approach is capable of select appropriate thresholds automatically and effectively, while it is shown to be less sensitive to noise and to perform better compared with other binarization algorithms.

The remainder of the paper is structured as follows: In Section 2, the NS approach is discussed in brief. Section 3 presents the previous work on binarization of historical documents of historical images and generation of ground truth images. In Section 4, we present our proposed hybrid method. Section 5 demonstrates experimental results. Finally, section 6 presents our conclusions and some directions for future research.

\section{Neutrosophic Theory: Reviews}

Neutrosophy [10;33] studies the nature, origin, and scope of neutralities. In neutrosophy theory, every object has not only a clear degree of the truth, but also a falsity degree and an indeterminacy degree [10].

NS provides a great tool for working with indeterminacy. For example, if A = "black", then Anti-A = "white", Non-A = "red, black, blue, yellow, etc." (Any color except white), and Neut - A = "blue, yellow, red, etc." (Any color except black and white) [12; 13].

\subsection{Neutrosophic sets}

A universe of discourse $U$, and a neutrosophic set $A$ included in $U$. An object $x$ in the set $M$ is described as $x(T, I, F) . T, I$ and $F$ are real standard or non-standard subsets of ]-0,1+ [ with $\sup T=t_{-} \sup , \inf T=t_{-} \inf , \sup I=i_{-} \sup$, $\inf I=i \inf , \sup F=f \sup , \inf F=f$ inf, $n$ sup $=t \_$sup $+i_{-} \sup +f_{-} \sup$, and $\inf F=f_{-} \inf , \bar{n}$ inf $=t$ inf $+\bar{i}$ inf $+\bar{i}$ inf. $T, I$ and $F$ are stated as neutrosophic components.

Where $x(T, I, F)$ belongs to $A$ in the following way: it is $i \%$ indeterminate, $t \%$ true in the set and $f \%$ false, where $i$ varies in $I, t$ varies in $T$, and $f$ varies in $F . T, I$, and $F$ are subsets, while $T, I$ and $F$ are operators / functions based on known or unknown parameters [10].

\subsection{Neutrosophic image}

A neutrosophic image $P_{N S}$ is presented by three subset $T, I$ and $F$. A pixel $P$ in the image is defined as $P(T, I, F)$ and fits to $W$ in the following way: it is $i \%$ indeterminate, $f \%$ false and $t \%$ true in the bright pixel set. The pixel $P(i, j)$ in the image domain is transformed into NS domain by;

$P_{N S}(i, j)=\{T(i, j), I(i, j), F(i, j)\}$

Where, $I(i, j), T(i, j)$ and $F(i, j)$ are the probabilities of belonging to indeterminate, the bright, and non-bright set, respectively, which are defined as; 


$$
\begin{aligned}
& T(i, j)=\frac{g(i, j)-g_{\text {min }}}{g_{\text {max }}-g_{\text {min }}} \\
& F(i, j)=1-T(i, j) \\
& I(i, j)=1-\frac{H o(i, j)-H o_{\text {min }}}{H o_{\text {max }}-H o_{\text {min }}} \\
& H o(i, j)=a b s(e(i, j))
\end{aligned}
$$

Which $H o(i, j)$ present the homogeneity value of $T$ at $(i, j)$, which is described by the local gradient value $e(i, j), g(i, j)$ is the intensity value of the pixel $(i, j), g_{\min }$ and $g_{\max }$ are the minimum and maximum value of $g(i, j)$ respectively $[12 ; 13]$.

\subsection{Entropy of neutrosophic image}

In any image the distribution of different gray levels is evaluated by using the entropy, the different intensities have equal probability and the intensities thus distribute uniformly if entropy is maximal. On the other hand, if the entropy is small, the intensities have different probabilities and their distributions are nonuniform.

In NS, The summation of the entropies of the three subsets $T, I$ and $F$ present neutrosophic image entropy and is employed to evaluate the distribution of the elements in the NS domain:

$$
\begin{aligned}
& E n_{N S}=E n_{T}+E n_{I}+E n_{F} \\
& E n_{T}=\sum P_{T}(i) \ln P_{T}(i) \\
& E n_{I}=\sum P_{I}(i) \ln P_{I}(i) \\
& E n_{F}=\sum P_{F}(i) \ln P_{F}(i)
\end{aligned}
$$

Where $E n_{T}, E n_{I}$ and $E n_{F}$ are the entropies of subsets $T, I$ and $F$, respectively, and $P_{T}(i), P_{I}(i)$ and $P_{F}(i)$ are the probabilities of element $i$ in $T, I$ and $F$, respectively. $E n_{T}$, and $E n_{F}$ are utilized to measure the distribution of the elements in the neutrosophic set, and $E n_{I}$ is employed to evaluate the indetermination distribution $[12 ; 13]$.

\section{$2.4 \lambda$-mean Operation}

The value of $I(i, j)$ is working to measure the indeterminate degree of $P_{N S}(i, j)$. To construct $I$ to be correlated with $F$ and $T$. Any changes in $T$ and $F$ influence the distribution of elements in $I$ and the entropy of $I[12 ; 13]$. In the gray level field, a $\lambda$-mean operation for image $X$ can be defined as;

$\bar{X}(i, j)=\frac{1}{w \times w} \sum_{m=i-w / 2}^{i+w / 2} \sum_{n=j-w / 2}^{j+w / 2} X(m, n)$

Where, $w$ is the local window size.

A $\lambda$-mean operation for $P_{N S}$ is defined as:

$$
\bar{P}_{N S}(\lambda)=P(\bar{T}(\lambda), \bar{I}(\lambda), \bar{F}(\lambda))
$$

with

$$
\begin{aligned}
& \bar{T}_{\lambda}(i, j)=\frac{1}{w \times w} \sum_{m=i-w / 2}^{i+w / 2} \sum_{n=j-w / 2}^{j+w / 2} T(m, n) \\
& \bar{F}_{\lambda}(i, j)=\frac{1}{w \times w} \sum_{m=i-w / 2}^{i+w / 2} \sum_{n=j-w / 2}^{j+w / 2} F(m, n)
\end{aligned}
$$

and

$\bar{I}_{\lambda}(i, j)=1-\frac{\overline{H o}(i, j)-\overline{H o}_{\min }}{\overline{H o}_{\max }-\overline{H o}_{\min }}$

Where, $\overline{H o}(i, j)$ is the homogeneity value of $\bar{T}(\lambda)_{\text {at }}(i, j)$.

Noise is removed and become more homogeneous in $T$ after the true subset $T$ is handled using the $\lambda$-mean operation $[12 ; 13]$.

\section{Previous Work}

Through this section two topics will be addresses; previous binarization techniques and their evaluation with respect to ground truth images.

\subsection{Binarizaiton of document images}

The problem of document image binarization can be solved using either global or local approaches. Global algorithms [e.g. Otsu [23]] select a single threshold value for the entire image. As many historical documents do not have a clear bimodal pattern, global thresholding 
is usually not a suitable approach for most of the historical document binarization [24]. On the other hand, Adaptive local thresholding which estimates a local threshold for each document image pixel is usually a better approach to handle the high variation associated with historical document images. During the last decade, there are a lot of proposed binarization algorithms that rely on adaptive approach [21]. To the best of our knowledge, none of the proposed methods can deal with all types of documents and degradations.

In a previous comparative works of $[22 ; 25 ; 26$; 27], they compare between different local adaptive algorithms for the binarization of grey images. Their experiments illustrated that Niblack's method [28] and its improved version by Sauvola's [8] gave best results among the local adaptive techniques tested. According to their findings, we select Sauvola's method as a first component of our proposed hybrid method. Sauvola's binarization technique is explained.

Sauvola's binarization technique is explained here in brief [22]. Consider a greyscale document image in which $g(x, y) \in[0,255]$ be the intensity of a pixel at location $(x, y)$. In local adaptive thresholding techniques, the aim is to compute a threshold $\mathrm{t}(\mathrm{x}, \mathrm{y})$ for each pixel such that

$$
o(x, y)=\left\{\begin{array}{cc}
0 & \text { if } g(x, y)<t(x, y) \\
255 \quad & \text { other wise }
\end{array}\right\}
$$

In Sauvola's binarization method [8], the threshold $t(x, y)$ is computed using the mean $m(x, y)$ and standard deviation $s(x, y)$ of the pixel intensities in a $\mathrm{w} \times \mathrm{w}$ window centered on the pixel $(x, y)$ :

$$
t(x, y)=m(x, y)\left[1+k\left(\frac{s(x, y)}{R}-1\right) \cdot\right.
$$

Where $R$ is the maximum value of the standard deviation ( $R=128$ for a greyscale document), and $\mathrm{k}$ is a parameter which takes positive values in the range $[0.2,0.5]$. The local mean $m(x, y)$ and standard deviation $s(x, y)$ adapt the value of the threshold according to the contrast in the local neighborhood of the pixel. When there is high contrast in some region of the image, $s(x, y) \approx R$ which results in $t(x, y) \approx m(x, y)$. This is the same result as in Niblack's method [28]. However, the difference comes in when the contrast in the local neighborhood is quite low. In that case the threshold $t(x, y)$ goes below the mean value thereby successfully removing the relatively dark regions of the background. The parameter $\mathrm{k}(\approx 0.34)[27,26]$ controls the value of the threshold in the local window such that the higher the value of $\mathrm{k}$, the lower the threshold from the local mean $m(x, y)$.

According to [29], Sauvola's method is shown to be more effective than Niblack's algorithm when the gray-level of the text is close to 0 and that of background close to 255. However, in images where the gray-levels of background and text pixels are close, the results are unsatisfactory. So, in this work, we combine Sauvola's method with neutrosophic approach to get improved performance for degraded document images.

\subsection{Ground truth generation}

In this section, several ground-truth generation tools illustrate [14]. For evaluate the performance of any binarization algorithm, we used ground truth. However, to create ground truth data is time-consuming, tedious and relying on formats [18]. These systems can work in many levels of granularity such as text lines, connected components, and individual pixels [15], it allows users to view a document image, zone it with simple mouse clicks, and specify information about each zone. With the help of "True- Viz" [16], the users can visualize and make ground truth and can create ground truth for words, glyphs and text lines together with their textual content.

In the PerfectDoc system [17], although it has the same qualification of [16], but it shows more advanced features such as; semantic annotations and multi-page reading order. Another system called PixLabeler [18]. It enables users to select groups of foreground pixels that has element with normal information and label them with one of a predefined set of labels. The combined several algorithms and suggested an online semiautomatic framework are addressed in [19], which is used for ground truth creation.

A relatively new scalable and robust system is presented in "Aletheia" [20]. The main advantage of this system is that, it's completely flexible, as it supports bottom-up as well as top-down ground truth generation schemes. 
Another web-based system for ground truth "WebGT" [14]. It supports real time collaboration among remote sites independent of the simple operating system and also gives several novel semi-automatic tools which have been mainly shown for annotating degraded documents. Lately, the "PhaseGT" [32] is a semi-automatic approach for ground truth generating.

The systems shown above cover different aspects of ground truth generation, and most of them have no assumptions upon the sort of documents (e.g. newspapers historical. etc.).

\section{Proposed Approach}

The proposed hybird binarization algorithm combines neutrosohpic theory and Sauvola's binarization techniques [8]. The various steps of the proposed algorithm is summarized in Figure 2.

\subsection{RGB to gray image conversion}

As preliminary step, the captured RGB image $\left(P_{R G B}\right)$ is converted to gray level images $(P G)$ using the NTSC standard method.

\subsection{Pre-processing phase}

A pre-processing stage play an important role for removing and reduce noise areas, smooth the background texture and enhanced highlight the contrast between background and foreground text areas [30]. The use of a lowpass Wiener filter has been proved efficient in this context [31], with the window size of the filter selected according to the minimum character line width [30]. In our proposed approach, the window size is selected as $3 \times 3$.

Finally the filtered gray level image $\left(P_{g w}\right)$ is ready now to be binarized in the next stages.

\subsection{Binarization}

We use a hybrid version of the NS thresholding method of [11]. The filtered gray image $\left(P_{g w}\right)$ is transformed into the neutrosophic domain, giving $\left(P_{N S}\right)$. Then, we use the $\lambda$-mean operation for reducing the indetermination degree of the image $\left(P_{N S}\right)$ which is evaluated by the entropy of the indeterminate subset. Thus, the image becomes more homogenous, uniform and more suitable to be thresholded. We then use the Sauvola's binarization method of [8] to obtain the binary image.

\subsection{Post-processing}

In this phase we employ the median filter with a window size of ${ }^{3 \times 3}$ to enhance the binary image for remove the noise which is output after adaptive thresholding process.

\subsection{Document analysis algorithm}

The proposed document analysis algorithm is summarized in Algorithm 1.

Algorithm 1 Proposed document analysis algorithm

1. Read in RGB image $P_{R G B}(x, y)$.

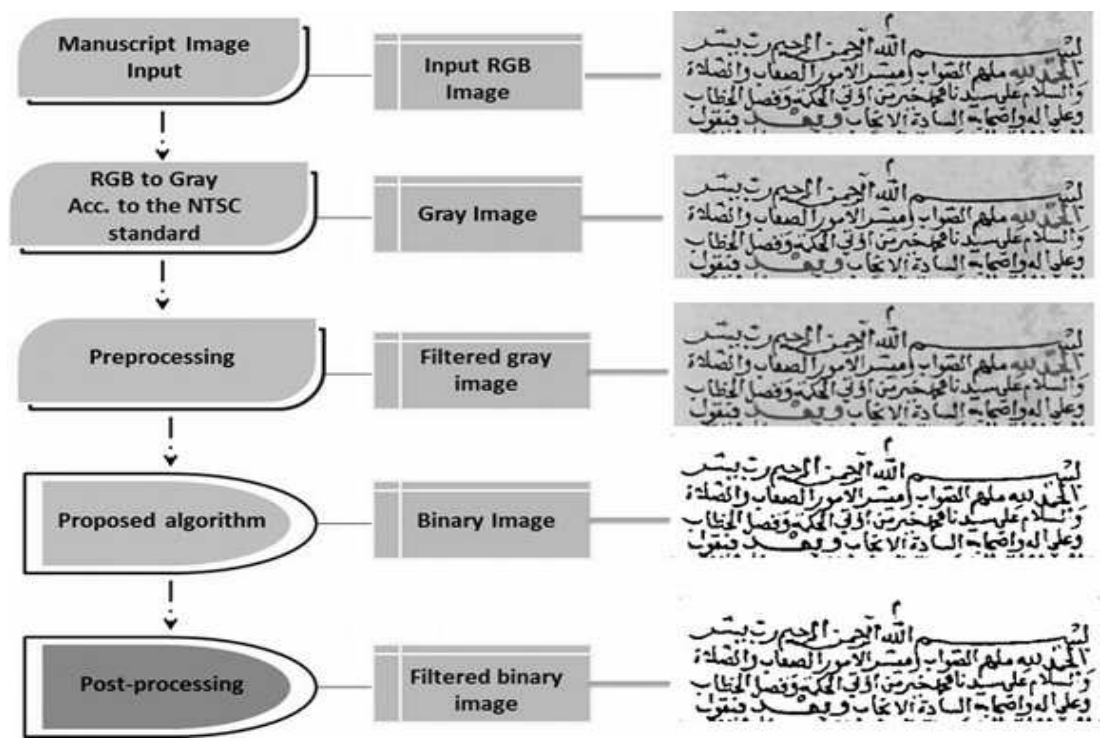

Figure 2. Overview of the proposed document analysis approach 
2. Convert to gray image $P_{g}(x, y)$ using NTSC standard.

3. Apply Wiener adaptive filter as preprocessing step to obtain $P_{g w}(x, y)$.

4. Transform $P_{g w}(x, y)$ into neutrosophic domain to obtain $P_{N S}(x, y)=$ $\{T(x, y), I(x, y), F(x, y)\} \quad$ according to the entropy of $P_{g w}(x, y)$ and its mean.

5. Measure the entropies of the three subsets $T, I$, and $F$.

6. Apply a $\lambda$-mean operation on $P_{N S}(x, y)$ to decrease its indetermination.

7. Segment the true subset $T$ using Sauvola's method [8].

8. Apply a median filter to remove noise as a post-processing step.

\section{Experimental Results}

In this section the proposed approach is applied on different degraded manuscript images from our data set. Then it's evaluated and compared with other binarization methods. We evaluate and assessed the performance of our proposed approach with those obtained by other binarization methods, namely Niblack's [28], Otsu's [23], Sauvola's [8], and Guo's [11].

\subsection{Data sets}

We focus on images that have several degradations such as multi-colored text lines, stains in the background, degraded characters and marks, and character diacritics. Our dataset contains (40) samples collected from both the database of $[3 ; 6]$ and from the electronic Arabic manuscripts of [5].

\subsection{Result}

For the experiments we used the Arabic handwritten images from three different source $[3 ; 6]$, each image contains one of the various degradations such as shadows, stains, nonuniform illumination, smudges, bleed-through, faint characters, as illustrated in Figure 1, and have various specifications, gray or color (24bit), jpeg or bmp format and their resolution range from 200 to $400 \mathrm{dpi}$, with the majority being at $300 \mathrm{dpi}$.
Three samples are shown in Figures 3, 4 and 5. The first sample for degraded manuscript image is shown in Figure 3.a. It has many problems such as; multicolored characters, stains and both side is visible. In the second sample image shown in Figure 4.a, it suffers from multicolored and distorted, strokes and low quality background. In the third sample shown in Figure 5.a, it suffers from several stains and appearance of both sides.

As can be seen in Figures 3.g, 4.g, and 5.g our algorithm outperforms the other approaches in terms of preservation of meaningful textual information. The other methods either fail to segment the foreground text, especially in the region of stains (Figures 3, 4 and 5 (d), (e), and (f), or segment foreground from background but add excessive noise (Figures 3, 4 and 5).

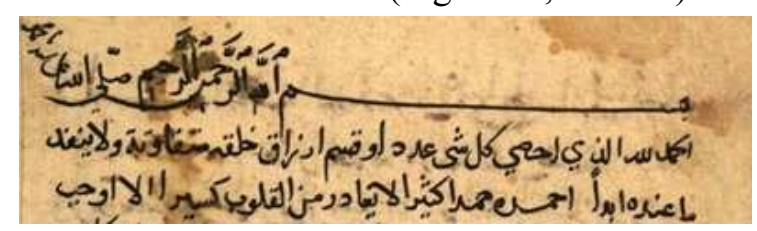

(a) original image

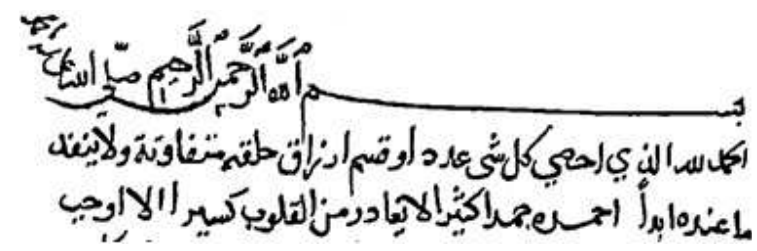

(b) ground truth image

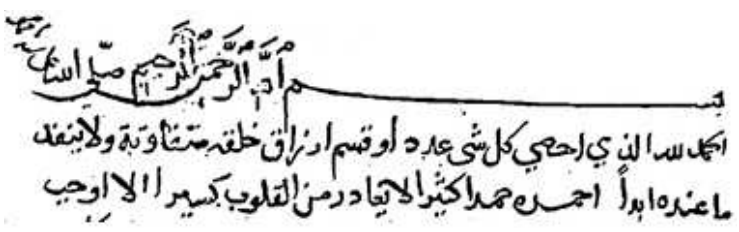

(c) Savoul's method

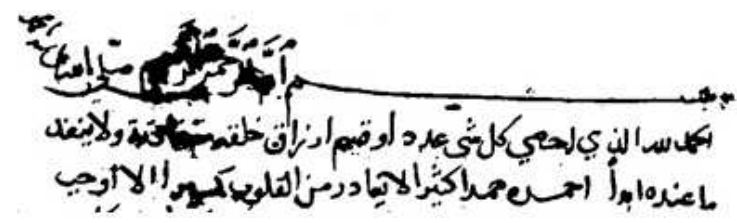

(d) Y-Guo method

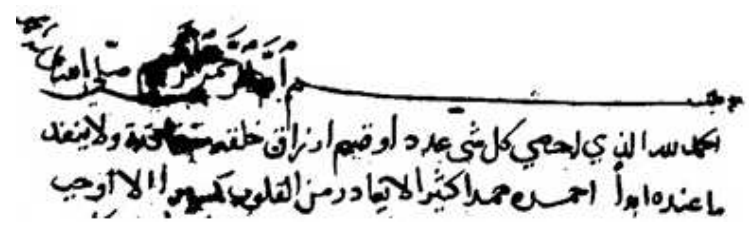

(e) Otsu's method 


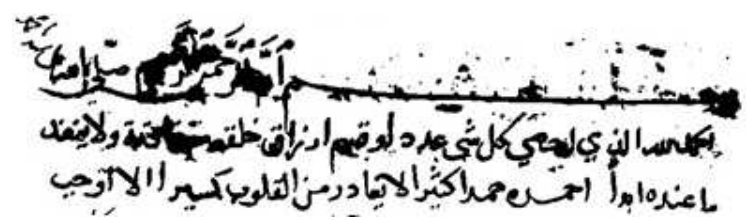

(f) Niblack's method

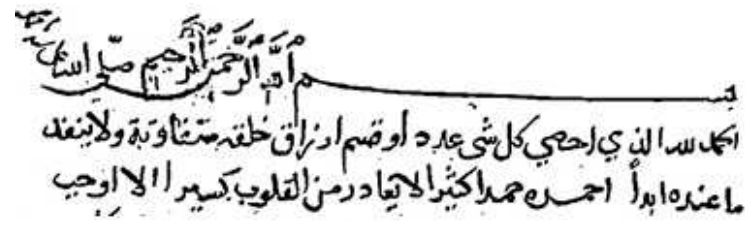

(g) proposed method

Figure 3. Visual comparison of the binarization algorithms

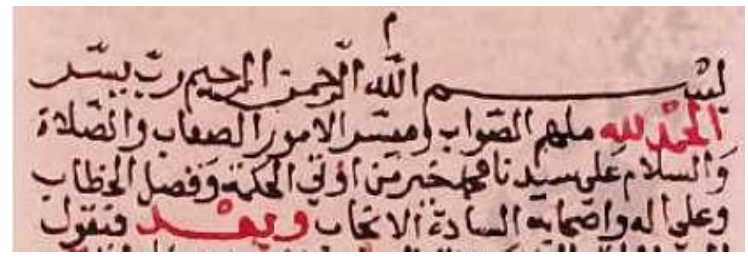

(a) original image

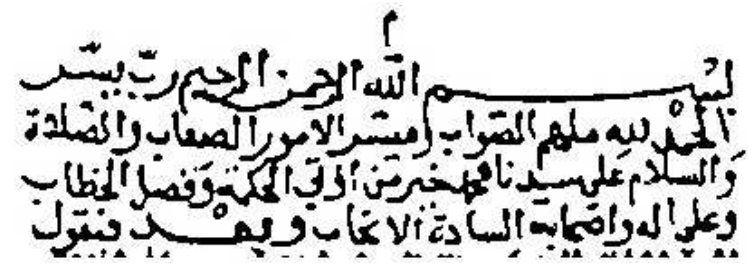

(b) ground truth image

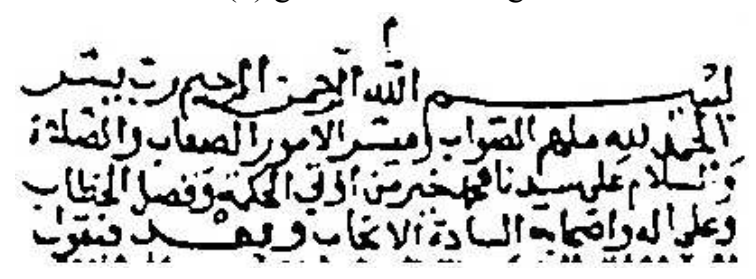

(c) Savoul's method

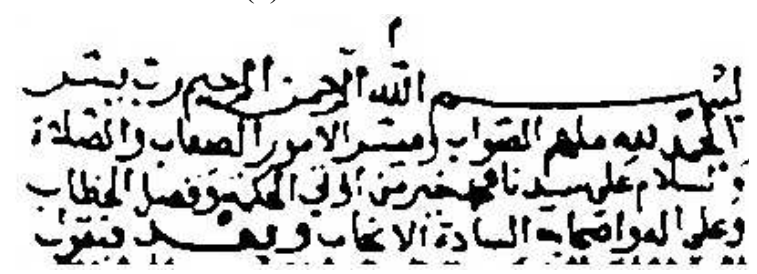

(d) Y-Guo method

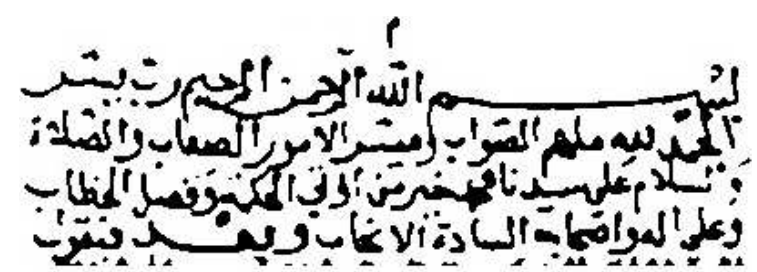

(e) Otsu's method

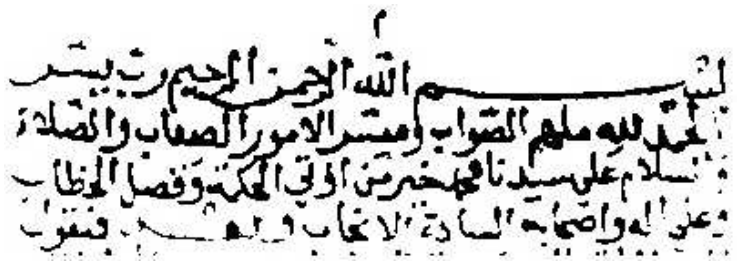

(f) Niblack's method

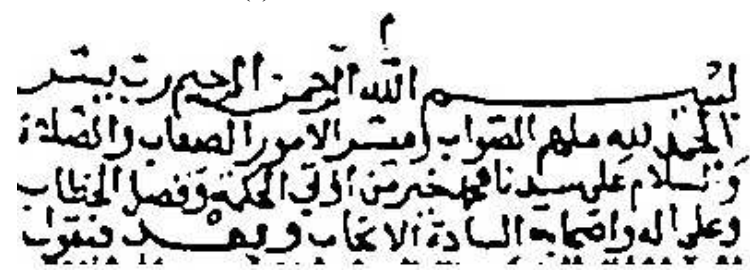

(g) proposed method

Figure 4. Visual comparison of the binarization algorithms

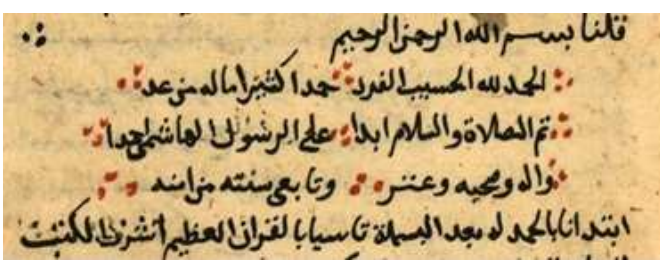

(a) original image

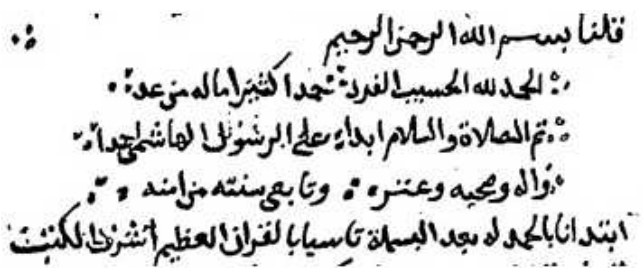

(b) ground truth image

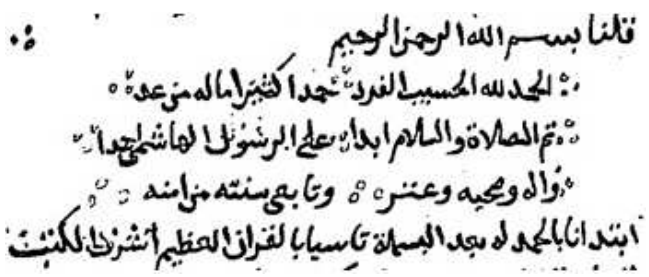

(c) Savoul's method 


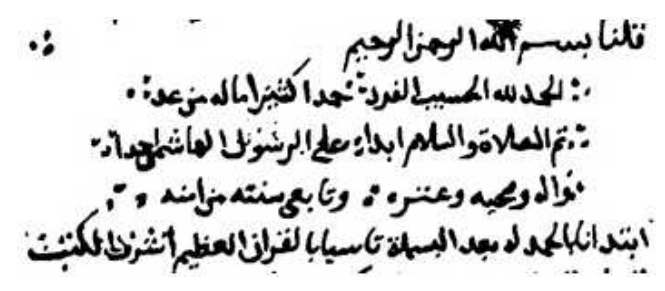

(d) Y-Guo method

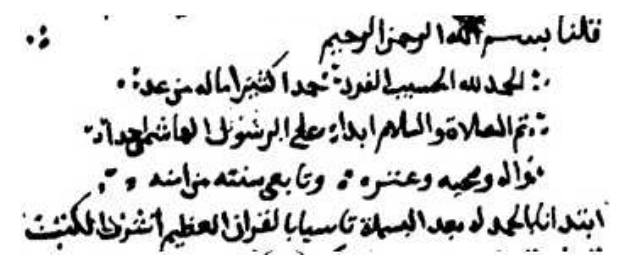

(e) Otsu's method

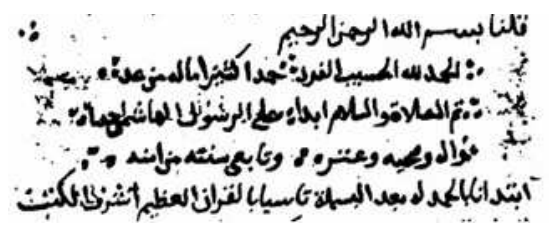

(f) Niblack's method

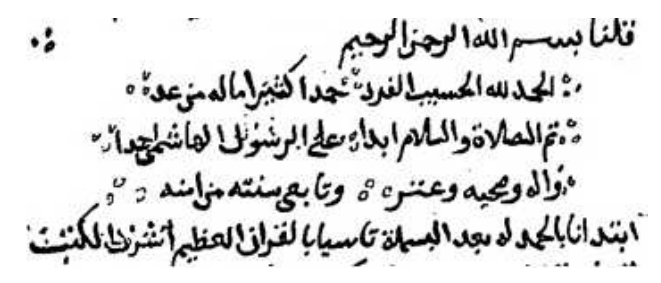

(g) proposed method

Figure 5. Visual comparison of the binarization algorithms

\subsection{Performance evaluation}

For evaluating the performance of our algorithm, several measures can be used [34; $35 ; 36]$, they are briefly addressed here:

\section{F-measure}

$F=\frac{2 \times \text { Precision } \times \text { Recall }}{\text { Precision }+ \text { Recall }}$

Recall $=\frac{T P}{T P+F N}$

Precision $=\frac{T P}{T P+F P}$

Which TN denotes true negatives; TP denotes true positives, FP false positive, and FN false negatives, respectively.
2. $F_{\beta}$-measure, defined as the weighted harmonic mean between precision and recall

$F_{\beta}=\frac{(1+\beta)^{2} \times T P}{(1+\beta)^{2} \times T P+\beta^{2} \times F N+F P}$

$\mathrm{MSE}=\frac{\sum\left[I_{1}(m, n)-I_{2}(m, n)\right]^{2}}{M \times N}$

Where $I_{1}$ and $I_{2}$ are the two images, and $M, N$ the image dimensions.

3. Negative Rate Metric (NRM), which is based on pixel wise mismatches between the binarized image and the ground truth [39], and joins the false negative rate $N_{F N}$ and the false positive rate $N_{F P}$ as;

$\mathrm{NRM}=\frac{\frac{N_{F N}}{N_{F N}+N_{T P}}+\frac{N_{F P}}{N_{F P}+N_{T N}}}{2}$

A good binarization quality is characterized by a lower NRM value.

4. Distance reciprocal distortion (DRD) metric, defined as:

$\mathrm{DRD}=\frac{\sum_{K=1}^{S} \mathrm{DRD}_{k}}{\mathrm{NUBN}}$

Where $\mathrm{DRD}_{k}$ is the distortion of the $k$-th flipped pixel and is computed using a $5 \times 5$ normalized weight matrix $W n_{m} \sim$ [29]. $\mathrm{DRD}_{k}$ equals the weighted sum of pixels in the $5 \times 5$ block of the ground truth GT that differ from the centered $k$-th flipped pixel at $(x, y)$ in the binarization result $B$.

$$
\begin{aligned}
\mathrm{DRD}_{k}= & \sum_{i=-2}^{2} \sum_{j=-2}^{2}\left|G T_{k}(i, j)-B_{k}(i, j)\right| \\
& \times W N_{m}(i, j)
\end{aligned}
$$

NUBN is the number of the non-uniform (not all white or black pixels) $8 \times 8$ blocks in the GT. 
5. Misclassification penalty metric (MPM). Measures the binarized image against the Ground Truth (GT),

$M P M=\frac{M P_{F N}+M P_{F P}}{2}$

whereMP $P_{F N}=\frac{\sum_{i=1}^{N_{F N}} d_{F N}^{i}}{D}, M P_{F P}=\frac{\sum_{j=1}^{N_{F P}} d_{F P}^{j}}{D}$

$d_{F N}^{i}$ and $d_{F P}^{j}$ denote the distance of the $i^{\text {th }}$ false negative and $j^{\text {th }}$ false positive pixel from the contour of the GT segmentation. The normalization factor $D$ is the sum over all the pixel-to-contour distances of the GT object. A low MPM score denotes that the algorithm is excellent at identifying an object's boundary [40].

Table 1 summarizes the results of the various binarization algorithms for a sample image, while Table 2 summarizes the results (averages over all dataset images) of the various binarization algorithms. Our proposed approach provides the best results with respect to all performance metrics.

Table 3 illustrates comparison of the performance of the proposed algorithm and the others against four printed image P04 which selected form DIBCO'9 P04, while Table 4 illustrates, performance of the proposed algorithm against selected image from DIBCO'09 and DIBCO'11 in term of F-measure, $\mathrm{F}_{\beta}$, Distance reciprocal distortion (DRD), Peak Signal to Noise Ratio (PSNR), Negative rate metric (NRM) and Misclassification penalty metric (MPM) respectively.

\subsection{Comparison between the proposed approach with the state of the art}

As explained above, we have tested and evaluated the proposed algorithm against the DIBCO series dataset, DIBCO'9, DIBCO'11 $[34 ; 40]$. Which consists of 10 ancient document images ( 5 are handwritten and 5 are machine printed), the images are of different sizes, 9 of them are gray, and one is in strokes by attaching the discontinuous parts, see Figure 3. Although the other binarization method provides acceptable results. In Table 2, we observe that the F-measure, $\mathrm{F}_{\beta}$, PSNR in the selected images is very high, this means achieve high performance. In order to obtain a more specific analysis of the performance of the proposed method, Tables 5, 6 provides the average of the F-measure, $F_{p s}$, PSNR, DRD, NRM, MPM values of various methods. From those tables, we found that the average Fmeasure of the printed image (90.9) is greater than the average F-measure of the handwritten images (87.85). The explanation for this is that most of the handwritten images are more degraded, and many parts of the texts have a low intensity, close to the intensity of the background. As is clear from the previous obtained result, our proposed algorithm provides the best results with respect to all performance metrics.

Table 1. Performance comparison of all binarization methods

\begin{tabular}{l|cccccc}
\hline Method & $\mathrm{F}$ & $\mathrm{F}_{\beta}$ & PSNR & DRD & NRM & MPM \\
\hline Guo (11) & 90.7 & 92.9 & 16.7 & 1.2 & 0.06 & 0.54 \\
Otsu's (23) & 90.8 & 93.1 & 16.8 & 1.1 & 0.06 & 0.55 \\
Niblack's (28) & 91.3 & 93.2 & 17.1 & 1.2 & 0.06 & 0.34 \\
Sauvola's (8) & 94.7 & 95.9 & 19.2 & 0.5 & 0.03 & 0.35 \\
proposed & 99.2 & 99.1 & 27.7 & 0.1 & 0.008 & 0.01 \\
\hline
\end{tabular}

Table 2. Performance in terms of F-measure, $F$ PSNR, DRD, NRM and MPM of the proposed algorithm using the selected dataset $[5 ; 6]$ included the average result.

\begin{tabular}{l|cccccc}
\hline Image & $\mathrm{F}-$ Measure & $\mathrm{F}_{\beta}$ & $\mathrm{DRD}$ & PSNR & $\mathrm{NRM}$ & $\mathrm{MPM}$ \\
\hline Image1 & 99.8 & 99.1 & 0.001 & 33.1 & 0.03 & 0.01 \\
Image2 & 84.3 & 90.7 & 0.08 & 13.5 & 4.02 & 2.5 \\
Image3 & 98.8 & 98.9 & 0.01 & 23.7 & 0.2 & 0.06 \\
Image4 & 99.2 & 99.2 & 0.007 & 26.6 & 0.3 & 0.14 \\
Image5 & 95.9 & 96.1 & 0.03 & 20.9 & 0.52 & 0.04 \\
Image6 & 99.2 & 99.1 & 0.008 & 27.1 & 0.11 & 0.01 \\
Image7 & 98 & 98.8 & 0.10 & 30.0 & 0.08 & 0.4 \\
Image8 & 97 & 97.8 & 0.9 & 22.6 & 0.09 & 3.3 \\
Image9 & 93.8 & 95.08 & 1.01 & 15.6 & 0.04 & 2.9 \\
Image10 & 93.5 & 94.6 & 1.1 & 16.2 & 0.05 & 1.1 \\
\hline Average & 95.9 & 96.9 & 0.32 & 22.9 & 0.54 & 1.04 \\
\hline
\end{tabular}

Table 3. Performance comparison of all binarization methods against P04 selected form DIBCO'9

\begin{tabular}{l|cccccc}
\hline Method & $\mathrm{F}$ & $\mathrm{F}_{p s}$ & PSNR & DRD & NRM & MPM \\
\hline Guo (11) & 82.5 & 83.9 & 13.7 & 3.8 & 0.04 & 9.1 \\
Otsu's (23) & 82.4 & 95.7 & 13.6 & 9.5 & 0.04 & 9.4 \\
Niblack's (28) & 76.6 & 77.8 & 12.1 & 13.7 & 0.05 & 23.1 \\
Sauvola's (8) & 88.04 & 88.4 & 16.4 & 9.5 & 0.1 & 0.23 \\
proposed & 92.8 & 97.3 & 18.4 & 2.3 & 0.05 & 0.2 \\
\hline
\end{tabular}

Table 4. Performance in terms of F-measure, $\mathrm{F}_{\beta}$, PSNR, DRD, NRM and MPM of the proposed algorithm using the Printed and Handwritten images of the DIBCO'09, and DIBCO'11 contests 


\begin{tabular}{l|cccccc}
\hline Image & F-Measure & $\mathrm{F}_{\beta}$ & PSNR & DRD & NRM & MPM \\
\hline P01 & 88.6 & 89.7 & 15.9 & 2.8 & 0.09 & 0.2 \\
P02 & 93.1 & 94.2 & 15.6 & 3.2 & 0.05 & 2.4 \\
P04 & 92.8 & 94.1 & 18.4 & 2.3 & 0.05 & 0.2 \\
P05 & 89.1 & 90.8 & 15.2 & 2.8 & 0.08 & 1.8 \\
\hline H02 & 89.4 & 95.0 & 23.3 & 3.9 & 0.04 & 0.1 \\
H03 & 85.0 & 87.3 & 15.7 & 4.1 & 0.11 & 0.4 \\
H04 & 87.0 & 88.1 & 17.6 & 4.0 & 0.10 & 0.1 \\
\hline HW1 & 87.5 & 87.9 & 19.8 & 3.1 & 0.10 & 0.04 \\
HW4 & 81.7 & 83.8 & 15.1 & 4.2 & 0.14 & 1.4 \\
HW5 & 92.5 & 93.7 & 17.9 & 2.3 & 0.05 & 1.2 \\
\hline
\end{tabular}

Table 5. Comparison of the performance of the proposed algorithm and the others against four printed image selected form DIBCO'9

\begin{tabular}{l|cccccc}
\hline Method & $\mathrm{F}$ & $\mathrm{F}_{p s}$ & PSNR & DRD & NRM & MPM \\
\hline Guo (11) & 89.8 & 91.85 & 16.05 & 0.03 & 3.55 & 4.16 \\
Otsu's (23) & 87.17 & 91.52 & 15.3 & 0.055 & 3.24 & 4.20 \\
Niblack's (28) & 82.87 & 84.5 & 13.20 & 0.05 & 15.22 & 7.9 \\
Sauvola's (8) & 87.85 & 91.5 & 15.17 & 0.05 & 6.64 & 5.8 \\
proposed & 90.9 & 94.87 & 16.27 & 0.06 & 1.15 & 2.8 \\
\hline
\end{tabular}

Table 6. Comparison of the performance of the proposed algorithm and the others against five handwritten image selected form DIBCO'9, DIBCO'11

\begin{tabular}{l|cccccc}
\hline Method & $\mathrm{F}$ & $\mathrm{F}_{p s}$ & PSNR & DRD & NRM & MPM \\
\hline Guo (11) & 67.90 & 68.60 & 12.65 & 26.55 & 0.08 & 42.31 \\
Otsu's (23) & 54.80 & 55.57 & 9.37 & 47.32 & 0.10 & 74.45 \\
Niblack's (28) & 62.07 & 62.85 & 10.26 & 31.45 & 0.07 & 39.93 \\
Sauvola's (8) & 84.21 & 89.95 & 15.42 & 4.33 & 0.13 & 0.87 \\
proposed & 87.13 & 91.37 & 17.70 & 3.63 & 0.08 & 0.77 \\
\hline
\end{tabular}

\subsection{Complexity and computational cost of the proposed algorithm}

The proposed binarization algorithm, implemented in Matlab (2009 a) takes 0.4 seconds on average for all images in the dataset on an Intel Core i3-2310- CPU@2.10 GHZ with 3GB RAMS 3.00 with Windows 7 . The complexity of the algorithm can be expressed as $O \frac{\left(n^{2} \times m^{2}\right)}{m^{2}}=O\left(n^{2}\right)$, where $\mathrm{n}$ is the size of the images and $m=2 d_{c}$ is the size of a grid patch.

\section{Conclusion and Future Work}

In this paper, we tackled the general problem of binarizing historical Arabic manuscript images. A lot of techniques used before, but no one between them demarcate to the others, especially for the old documents images having different

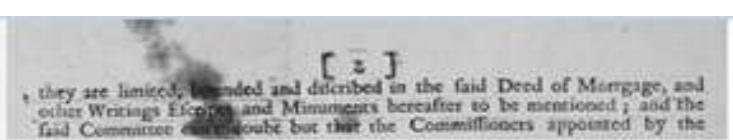

(a)

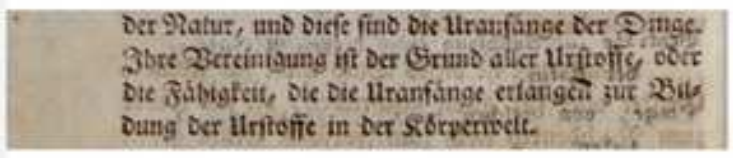

(b)

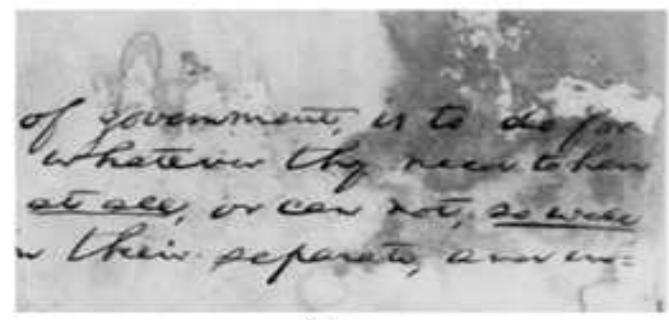

(c)

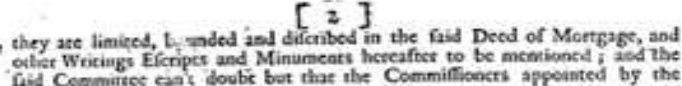

(d)

Orr \$2atus, uno Drfe find oic Utranfänge Der Dinge.

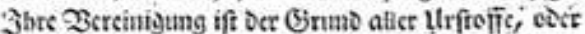

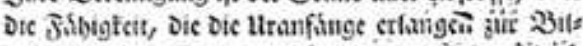
Dumg Der ttritefic in Der Solvernelt.

(e)

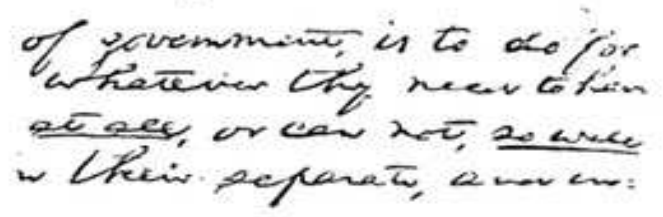

(f)

Figure 6. Visual comparison of the proposed binarization approach. Result selected from DEBICO series competition: $(a, b, c)$ original image; $(\mathrm{d}, \mathrm{e}, \mathrm{f})$ proposed approach result.

degradation characteristics. We presented a novel hybird technique based on neutrosphic theory and savoula's technique. Extensive experiments on selected database have demonstrated the effectiveness and robustness of the proposed method. As a future work, the parameter of our algorithm needs to be optimized for other degradation types of all documents. 


\section{REFERENCES}

1. NAFCHI, H., R. F. MOGHADDAM, M. CHERIET, Phase-Based Binarization of Ancient Document Images: Model and Applications, IEEE Trans. on Image Processing. vol. 23(7), 2014, pp. 2916-2930.

2. NTIROGIANNIS, K., B. GATOS, I. PRATIKAKIS, Performance Evaluation Methodology for Historical Document Image Binarization. IEEE Trans. on Image Processing. vol. 22(2), 2013, pp. 595-609.

3. AMIN, K. M., M. A. AHMAD, A. ALI, A Novel Binarization Algorithm for Historical Arabic Manuscripts using Wavelet Denoising, Int. J. of Computing and Inf. Sciences. Vol. 13(1), 2013.

4. AMIN, K. M., M. ElFATTAH, A. E. HASSANIEN, G. SCHAEFER, A Binarization Algorithm for Historical Arabic Manuscript Images using a Neutrosophic Approach. In Computer Engineering Systems (ICCES), 9th Intl. Conference, pp. 266-270, 2014.

5. http://www.wqf.me.comlast accessed at 9 P.M, 10 Jan 2015.

6. http://ocp.hul.harvard.edu/ihp/manuscripts. html, last accessed at 9 P.M, 10 Sep. 2015.

7. P. STATHIS, E. KAVALLIERATOU, N. PAPAMARKOS, An Evaluation Technique for Binarization Algorithms, Journal of Universal Computer Science, vol. 14(18), 2008, pp. 3011-3030.

8. SAUVOLA, J., M. PIETIKAINEN, Adaptive Document Image Binarization, Pattern Recognition, vol. 33(2), 2000, pp. 225-236.

9. MOHAN, J., V. KRISHNAVENI, Y. GUO, A New Neutrosophic Approach of Wiener Filtering for MRI Denoising, Measurement Science Review, Vol. 13(4), 2013, pp. 177-168.

10. SAMARANDACHE, F., A Unifying Field in Logics: Neutrosophic Logic. Neutrosophy, Neutrosophic Set, Neutrosophic Probability, (third edition). American Research Press, 2003.

11. Y. CHENG, HENG-DA, Y. GUO, A New Neutrosophic Approach To Image
Thresholding, New Mathematics and Natural Computation, vol. 4(3), 2008, pp. 291-308.

12. GUO, Y., H. D. CHENGA, New Neutrosophic Approach To Image Segmentation, Advances in Multimedia, Vol. 42(5), pp. 587-595, 2009.

13. ZHANG, M., Novel Approaches to Image Segmentation Based on Neutrosophic Logic, Doctoral Dissertation, Utah State University, 2010.

14. BILLER, OFER, WebGT: An Interactive Web-Based System for Historical Document Ground Truth Generation. ICDAR, pp. 305-308. 2013.

15. YANIKOGLU, B., L. VINCENT. Pink Panther: A Complete Environment for Ground Truthing and Benchmarking Document Page Segmentation, Pattern Recognition. vol. 31(9), 1998, pp. 1191-1204.

16. LEE, H., CHANG, T. KANUNGO. The Architecture of TRUEVIZ: A groundTRUth/metadata Editing and VIsualiZing toolkit. Pattern recognition. Vol. 36(3), pp. 811-825, 2003.

17. YACOUB, S., S. VINAY, S. S. NUSRULLA. Perfectdoc: A Ground Truthing Environment for Complex Documents. In: Document Analysis and Recognition, 2005. Proceedings. Eighth International Conference on. IEEE, 2005, pp. 452-456.

18. SAUND, E., J. LIN, P. SARKAR, PIXLABELER: User Interface for Pixellevel Labeling of Elements in Document Images. In: Document Analysis and Recognition, 2009. ICDAR'09. 10th Intl. Conf. on. IEEE, 2009, pp. 646-650.

19. FISCHER, A., Ground Truth Creation for Handwriting Recognition in Historical Documents. Proceedings of the 9th IAPR Intl. Workshop on Document Analysis Systems. ACM, 2010, p. 310.

20. CLAUSNER, C., S. PLETSCHACHER, A. ANTONACOPOULOS, Aletheia-an advanced document layout and text ground-truthing system for production environments. International Conference on In Document Analysis and Recognition (ICDAR), pp. 48-52, 2011. 
21. FENG, M.-L., Y.-P. TAN, Adaptive Binarization Method for Document Image Analysis, IEEE on Multimedia and Expo, Vol. 1, pp. 339-342, 2004.

22. SHAFAIT, F., KEYSERS, D., BREUEL, T. $M$. E_cient implementation of local adaptive thresholding techniques using integral images. In Electronic Imaging. International Society for Optics and Photonics, pp. 681510-681510, 2008.

23. OTSU, N., A Threshold Selection Method from Gray-Level Histograms, IEEE Transactions on Systems, Man and Cybernetics, Vol. 9(1), pp. 62-66, 1979.

24. SU, B. S. LU, C. L. TAN, Binarization of historical handwriting document images using local maximum and minimum filter, in Proc. Int. Workshop Document Anal. Syst., pp. 159-166, 2010.

25. TRIER, O. D., T. TAXT, Evaluation of binarization methods for document images, IEEE Trans. On Pattern Analysis and Machine Intelligence 17, pp. 312-315, March 1995.

26. BADEKAS, E., N. PAPAMARKOS, Automatic evaluation of document binarization results, in 10th Iberoamerican Congress on Pattern Recognition, pp. 1005-1014, [Havana, Cuba], 2005.

27. SEZGIN, M., B. SANKUR, Survey Over Image Thresholding Techniques and Quantitative Performance Evaluation, Journal of Electronic Imaging, vol. (13)1, pp. 146-165, 2004.

28. NIBLACK, W., An Introduction to Digital Image Processing, Prentice Hall, Englewood Cli_s, 1986.

29. KHURRAM, K., Comparison of Niblack inspired Binarization methods for ancient documents, IST/SPIE Electronic Imaging, pp. 72470U- 72470U, 2009.

30. GATOS, B. I. PRATIKAKIS, S. PERANTONIS, An Adaptive Binarization Technique for Low Quality Historical Documents, DAS 2004, LNCS 3163, pp. 102-113, 2004.
31. JAIN, A., Fundamentals of Digital Image Processing, Pr. Hall, 1989.

32. NAFCHI, H., S. AYATOLLAHI, R. FARRAHI, M. CHERIET, An efficient ground truthing tool for binarization of historical manuscripts, $12^{\text {th }}$ Int. Conf. on Document Analysis and Recognition, pp. 807-811, 2013

33. SMARANDACHE, F., A Unifying Field in Logics Neutrosophic Logic. Neutrosophy, Neutrosophic Set, Neutrosophic Probability, 3rd Ed., American Research Press, 2003.

34. GATOS, B., K. NTIROGIANNIS, I. PRATIKAKIS, ICDAR 2009 document image binarization contest (DIBCO 2009), Int. Conf. on Document Analysis and Recognition, pp. 1375-1382, 2009.

35. LU, H., A. C. KOT, Y. Q. SHI, DistanceReciprocal Distortion Measure for Binary Document Images, IEEE Signal Processing Letters, vol. 11(2), pp. 228-231.

36. SU, B., S. LU, C. L. TAN. Robust document image binarization technique for degraded document images. Image Processing, IEEE Transactions on. Vol. (22)4, pp. 1408-1417, 2013.

37. MAYNARD, D., W. PETERS, Y. LI. Metrics for evaluation of ontology-based information extraction. International World Wide Web conference, 2006.

38. LU, H., A. C. KOT, Y. Q. SHI, Distancereciprocal distortion measure for binary document images, IEEE Signal Processing Letters, Vol. 11(2), pp. 228-231, 2004.

39. AGUILERA, J., H. WILDENAUER, M. KAMPEL, M. BORG, D. THIRDE，J. FERRYMAN, Evaluation of motion segmentation quality for aircraft activity surveillance, 2nd Joint IEEE Int.Workshop on Visual Surveillance and Performance Evaluation of Tracking Surveillance, pp. 293- 300, 2005.

40. PRATIKAKIS, I. B. GATOS, K. NTIROGIANNIS, ICDAR 2011 Document Image Binarization Contest, Intl. Conf. on Document Analysis and Recognition, pp. 1506-1510, 2011. 